\title{
Interactive comment on "On the need of a time and location dependent estimation of the NDSI threshold value for reducing existing uncertainties in snow cover maps at different scales" by Stefan Härer et al.
}

\section{S. Gascoin (Referee) \\ simon.gascoin@cesbio.cnes.fr}

Received and published: 2 November 2017

The NDSI is a spectral index that is commonly used to map the snow cover extent from optical multispectral satellite imagery. Here, the authors evaluated the stability of the NDSI threshold to map the snow cover in two alpine sites from a time series of Landsat images. The paper reads rather well. I found that the wording is sometimes a bit awkward but I'm not an expert in English grammar! 
results to demonstrate the added-value of this paper with respect to a previous paper by the same authors (Härer et al. 2016 GMD). I made some suggestions below. I hope the authors will find my comments useful.

1) The authors choose not to apply atmospheric correction to the Landsat scenes. Absorption and scattering by the air molecules and aerosols can have an impact on the NDSI, although atmospheric absorbance is typically low in the SWIR and Green wavelengths, which are used to compute the NDSI (especially in high elevation areas). However, this shall be better discussed since the authors justify their study by the fact that the 0.4 NDSI threshold is used for the MODIS snow products ("This is of special interest as MODIS snow cover products are today the most frequently applied satellite snow cover maps"). If the authors referred to NASA's MOD10/MYD10 snow products, the atmospheric correction is applied before computing the NDSI. In addition, the NDSI threshold is not applied anymore in the latest collection 6. More importantly, the lack of atmospheric correction cast doubts on the significance and the transferability to other sites of the "newly developed calibrated quadratic polynomial model which is accounting for seasonal threshold dynamics". The authors should clarify this to avoid confusing readers who are not familiar with satellite imagery processing.

2) The authors give too much details about the PRACTISE software, which was used to rectify the photographs from the time lapse cameras, whereas it was already described in another journal (for instance Fig. 2 was already published in Härer et al. 2016; Fig. 3 and Fig. 4 further illustrate the PRACTISE workflow and are not useful in my opinion). An important step for this study is rather how these camera snow maps were resampled to the Landsat resolution and it is missing. Indeed, camera snow maps have a submeter ground sampling distance. As a result, it is likely that some Landsat pixels were classified as "snow" from the camera images, while they were actually not $100 \%$ snow covered in the camera images.

3) The literature review in the introduction was a bit overlooked. The authors state that "The used snow-cover mapping approaches can be grouped into three categories:

Printer-friendly version

Discussion paper 
manual interpretation, classification-based, and index-based methods" but there are other approaches based on spectral unmixing. The proposed "geology dependent offset" is the result of a well-known phenomena (e.g. Kaufman et al. 2002, GRL), and is similar to the NDSI_0 method developed by Chaponnière et al. (2005, IJRS); it can be also seen as an extreme simplification of a spectral mixture analysis used in other MODIS snow products (e.g., Sirguey et al., 2009 RSE; Painter et al., 2009 RSE).

4) Overlap with Härer et al. (2016 GMD). In a previous paper, the authors already showed the results of the NDSI threshold calibration on three Landsat scenes using the same method. Here, the authors extend this approach to a time series of Landsat images, which is a good idea I think. The authors obtain a (weak) seasonal cycle in the calibrated NDSI threshold value. Given that an important insight of this TCD paper was already introduced by Härer et al. 2016 ("A spatial and temporal adjustment of NDSI thresholds is therefore important to ensure optimum results in the snow cover mapping"), I do not think that the "investigation of the reasons of this effect is beyond this study". The authors could test if the calibrated NDSI_tr is correlated to the solar zenith and azimuth angles. In addition, the authors did not consider the hypothesis that this seasonal cycle may be due to inaccurate snow detection in the camera images. What is the bit depth of the camera images? Snow detection from terrestrial camera imagery is difficult in shaded slopes especially from 8-bits RGB pictures. The reported accuracy (below 5\% misclassified pixels from visual inspection) can lead to significant changes in the NDSI, which are probably within the range of the calibrated NDSI threshold variability? This could be tested by excluding shaded areas before computing the accuracy of the Landsat snow masks. Another source of error that was not discussed is the one due to the geometric distortion between oblique images and nadir-looking satellite images.

Printer-friendly version

Specific comments

P1L12: Earth not earth 
P3L22: glacierized not glaciered

P3L23-25: It could be useful to show the spectral profiles of the snow-free substratum (limestone is more reflective in the visible range than gneiss).

P4L13: I do not think that this statement is true "no atmospheric correction is applied (..) the majority of studies that apply the NDSI for snow cover mapping". Many studies use the MOD10 snow products, or TMSCAG for Landsat, which use surface reflectances.

P3L17: NDSI and NDSI_thr are written in equation mode, sometimes in plain text.

P4L9: photographs not photographies.

What is the acquisition time of the camera? is it synchronous to Landsat overpass time?

P4L31: did you find a difference in the results between Landsat 8 and the Landsat $5 / 7$ ? Landsat 8 instrument has higher radiometric resolution which improves snow classification in mountains (less saturation, higher SNR in shaded slopes).

P6L8: Note that this metric is usually referred to as accuracy and may not be a robust performance measure when the number of a class is much greater than the number of the other one.

P7L29: if vertical, these rock faces are not visible in images captured by nadir-looking sensors like Landsat 7.

P7L31: "NDSI reflectances" does not make sense

P8L10-12: this sentence is not clear to me.

P8L31: fitted against what? Day of year I think.

Printer-friendly version

P9L18: why not using real MODIS images instead?

P9L25: if I understand well, the resampling to $500 \mathrm{~m}$ has increased the optimal threshold value. Can you think of an explanation? 
Fig. 5: rainbow colormaps are not recommanded (Borland 2007). I am also surprised by the choice of the projection (plate carrée? non-equidistant projections are not recommended for this kind of maps)

Interactive comment on The Cryosphere Discuss., https://doi.org/10.5194/tc-2017-177, 2017. 